\title{
The impact of high speed machining on computing and automation
}

\begin{abstract}
Machine tool technologies, especially Computer Numerical Control (CNC) High Speed Machining (HSM) have emerged as effective mechanisms for Rapid Tooling and Manufacturing applications. These new technologies are attractive for competitive manufacturing because of their technical advantages, i.e. a significant reduction in lead-time, high product accuracy, and good surface finish. However, HSM not only stimulates advancements in cutting tools and materials, it also demands increasingly sophisticated $\mathrm{CAD} / \mathrm{CAM}$ software, and powerful $\mathrm{CNC}$ controllers that require more support technologies. This paper explores the computational requirement and impact of HSM on CNC controller, wear detection, look ahead programming, simulation, and tool management.
\end{abstract}

Keyword: High speed machining; Look-ahead; Automation; Simulation 